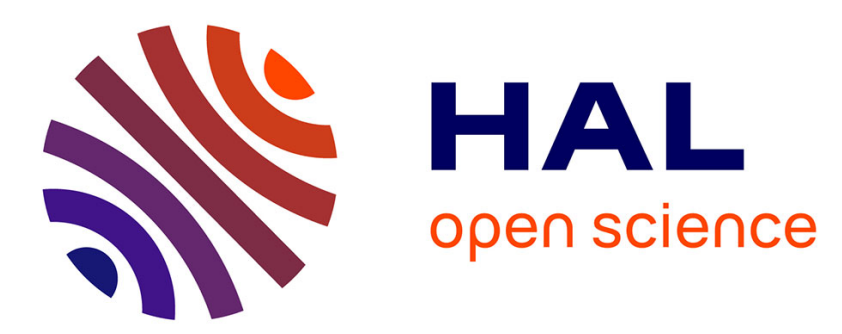

\title{
Parallel Transport with Pole Ladder: Application to Deformations of time Series of Images \\ Marco Lorenzi, Xavier Pennec
}

\section{To cite this version:}

Marco Lorenzi, Xavier Pennec. Parallel Transport with Pole Ladder: Application to Deformations of time Series of Images. GSI2013 - Geometric Science of Information, Aug 2013, Paris, France. pp.68-75, 10.1007/978-3-642-40020-9_6. hal-00819898

\section{HAL Id: hal-00819898 \\ https://hal.inria.fr/hal-00819898}

Submitted on 21 Oct 2013

HAL is a multi-disciplinary open access archive for the deposit and dissemination of scientific research documents, whether they are published or not. The documents may come from teaching and research institutions in France or abroad, or from public or private research centers.
L'archive ouverte pluridisciplinaire HAL, est destinée au dépôt et à la diffusion de documents scientifiques de niveau recherche, publiés ou non, émanant des établissements d'enseignement et de recherche français ou étrangers, des laboratoires publics ou privés. 


\title{
Parallel Transport with Pole Ladder: Application to Deformations of time Series of Images
}

\author{
Lorenzi Marco ${ }^{1}$ and Xavier Pennec ${ }^{1}$ for $\mathrm{ADNI}^{\star}$ \\ Project Team Asclepios, INRIA Sophia Antipolis, France **
}

\begin{abstract}
Group-wise analysis of time series of images requires to compare observed longitudinal evolutions. In medical imaging, longitudinal anatomical changes can be modeled by using deformations resulting from the non-rigid registration of follow-up images. The comparison of longitudinal trajectories is therefore the transport of longitudinal deformations in a common reference frame. We previously showed that the Schild's Ladder is an efficient and simple method for the parallel transport of diffeomorphic deformations parameterized by tangent velocity fields. The Schild's Ladder is based on the construction of a geodesic parallelogram. The base vertices of the parallelogram are the pairs of follow-up images and another vertex is the reference frame. By building the geodesic diagonals of the parallelogram, Schild's Ladder computes the missing vertex which corresponds to the transported follow-up image. However, Schild's Ladder may be inefficient in case of time series of multiple time points, in which the computation of the geodesic diagonals is required several times. In this paper we propose a new algorithm, the Pole Ladder, in which one diagonal of the parallelogram is the baseline-to-reference frame geodesic. This way we have to compute only one diagonal for each time point along the curve. In this work we show that the transport of the Pole ladder and the Schild's Ladder are equivalent. Moreover, we show how the Pole ladder can be succesfully applied to the clinical problem of the measurement of the longitudinal atrophy progression in the brain for a group of patients affected by Alzheimer's disease.
\end{abstract}

\section{Introduction}

Group-wise analysis of time series of images requires to compare observed longitudinal evolutions. Non-rigid registration is one of the main instruments for modeling anatomical differences from images. The aim of non-rigid registration

\footnotetext{
* Data used in preparation of this article were obtained from the Alzheimer's Disease Neuroimaging Initiative (ADNI) database (www.loni.ucla.edu/ADNI). As such, the investigators within the ADNI contributed to the design and implementation of ADNI and/or provided data but did not participate in analysis or writing of this report. A complete listing of ADNI investigators can be found at:www.loni.ucla.edu/ADNI/Collaboration/ADNI_Authorship_list.pdf

** This work was partially funded by the European Research Council (ERC advanced Grant MedYMA), ANR blanc Karametria and the EU project Care4Me.
} 
is to encode the observed structural changes as deformation fields densely represented in the image space.

We can identify two distinct settings for the application of non-rigid registration: longitudinal and cross-sectional. In the former, non-rigid registration estimates the deformation field which explains the intra-subject longitudinal anatomical changes. In the latter, the deformation field accounts for the anatomical differences between different subjects (inter-subject), in order to match homologous anatomical regions. These two settings deeply differ, since the deformations of the cross-sectional setting are often a scale of magnitude higher than the ones characterizing the subtle variations of the longitudinal setting.

In case of group-wise analysis of longitudinal deformations, longitudinal and cross-sectional settings must be integrated in a consistent manner. More precisely, we want to normalize the baseline to follow-up deformation in a common reference frame, by means of inter-subject registration. This way, group-wise analysis of longitudinal deformations can be performed in the reference space.

Parallel Transport in Diffeomorphic Registration. Among the different normalization methods, the parallel transport of longitudinal deformations is a powerful and promising tool which can be used within the "diffeomorphic registration" setting. Mathematically, parallel transporting a vector $X$ along a curve $\gamma(t)$ consists in translating it across the tangent spaces to the curve by preserving its parallelism, according to a given derivative operation called (affine) connection, and indicated with $\nabla_{\gamma(t)}(X)$.

Two main approaches are currently pursued for diffeomorphic registration. In the former, called "Large Deformation Diffeomorphic Metric Mapping (LDDMM)" $[112$ the deformation is the endpoint of a Riemannian geodesic parameterized by time-varying velocity fields. We recall the Riemannian geodesics are defined with respect to the Levi-Civita connection, which is the unique torsionfree connection which preserves the metric" In the latter, called "Stationary Velocity Field" setting [1, the deformation is the endpoint of the one-parameter subgroup parameterized by a stationary velocity field (SVF). One parameters soubgroups are non-metric geodescis with respect to the Cartan-Shouten connections [10]. Within the diffeomorphic registration, the transport of longitudinal deformations can therefore be naturally formulated as the parallel transport of tangent vectors along geodesics according to the underlying connection.

Schild's Ladder for the Parallel Transport of Longitudinal Trajectories. The Schild's Ladder is a method for infinitesimally transporting a vector along a given curve through the construction of geodesic parallelograms, and provides a straightforward method to compute a first order approximation of the parallel transport of a vector along a curve using geodesics only [95]. In [7] we proposed the Schild's Ladder for the parallel transport of longitudinal deformations parameterized by tangent vectors, and we provided a closed form formula

\footnotetext{
${ }^{1}$ For a more detailed discussion we refer to classical books on Riemannian geometry, for example to [3].
} 
for its application in the SVFs setting. This enabled effective and straightforward transport of longitudinal deformations of synthetic and real images. In spite of its simple formulation, Schild's Ladder may be however computationally inefficient in case of transport time series of multiple time points, in which the computation of the geodesic diagonals of the parallelogram is required several times.

\subsection{Contributions and Paper's Organization}

In this paper we propose the Pole Ladder, a new simplified algorithm for the parallel transport of tangent vectors along arcs of geodesics. The Pole Ladder is still based on the construction of geodesic parallelograms, and we show that it is mathematically equivalent to the Schild's Ladder. Moreover, the Pole Ladder has the advantage of minimizing the number of estimations of geodesics when applied to the parallel transport of time series of deformations.

We introduce and illustrate the computational advantages of the Pole Ladder in Section 2. In Section 3 we demonstrate its succesfull application to the clinical problem of the modeling of the longitudinal atrophy progression in the brain for a group of patients affected by Alzheimer's disease.

\section{Pole Ladder for the Parallel Transport of Longitudinal Deformations}

Before introducing the Pole Ladder scheme, we recall here the principles of Schild's Ladder for transporting longitudinal deformations.

The Schild's Ladder. Let $M$ a manifold and $C$ a curve parametrized by the parameter $\tau$ with $\left.\frac{\partial C}{\partial \tau}\right|_{T_{0}}=\mathbf{u}$, and $\mathbf{A} \in T_{P_{0}} M$, a tangent vector on the curve at the point $P_{0}=C(0)$. Let $P_{1}$ be a point on the curve relatively close to $P_{0}$, i.e. separated by a sufficiently small parameter value $\tau$. The Schild's Ladder computes the parallel transport of $\mathbf{A}$ along the curve $C$ as follows (Figure 1 ):

1. Define a curve on the manifold parametrized by a parameter $\sigma$ passing through the point $P_{0}$ with tangent vector $\left.\frac{\partial}{\partial \sigma}\right|_{P_{0}}=\mathbf{A}$. Chose a point $P_{2}$ on the curve separated by $P_{0}$ by the value of the parameters $\sigma$. The values of the parameters $\sigma$ and $\tau$ should be chosen in order to construct the Schild's Ladder within a single coordinate neighborhood.

2. Let $l$ be the geodesic connecting $P_{2}=l(0)$ and $P_{1}=l(\lambda)$, we chose the "middle point" $P_{3}=l(\lambda / 2)$. Now, let us define the geodesic $r$ connecting the starting point $P_{0}$ and $P_{3}$ parametrized by $\rho$ such that $P_{3}=r(\rho)$. Extending the geodesic at the parameter $2 \rho$ we reach the point $P_{4}$. We can now pick a curve connecting $P_{1}$ and $P_{4}$. The vector $A^{\prime}$ tangent to the curve at the point $P 1$ is the parallel translation of $A$ along $C$.

3. If the distance between the points $P_{0}$ and $P_{1}$ is large, the above construction can be iterated for a sufficient number of small steps. 


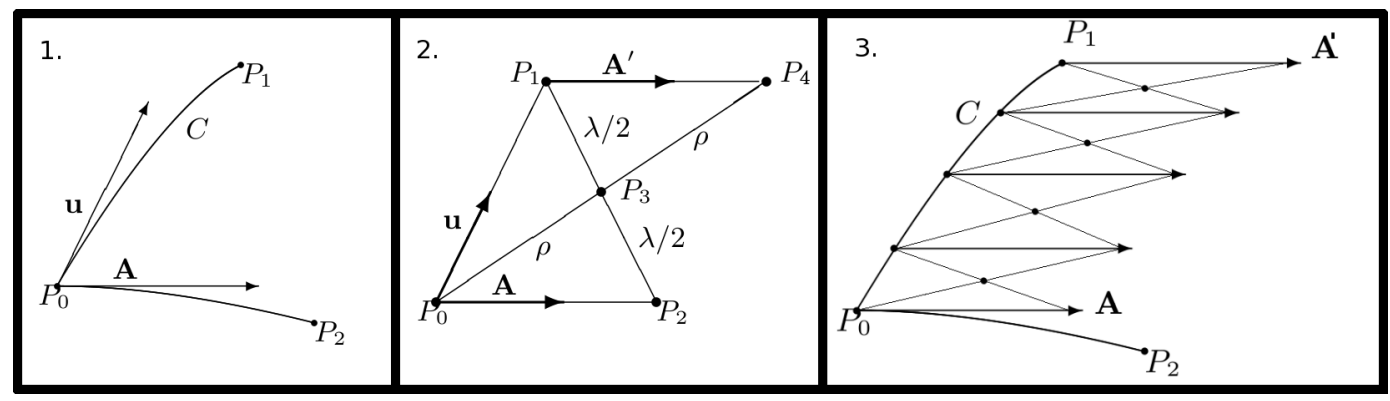

Fig. 1: The Schild' ladder parallel transports a vector $\mathbf{A}$ along the curve $C$ by iterative construction of geodesic parallelograms.

The algorithmic interest of the Schild's ladder is that it only relies on the geometrical information encoded by the geodesics. Although the geodesics on the manifold are not sufficient to recover all the information about the space properties, such as the torsion of the connection, it has been shown that the Schild's Ladder describes the parallel transport with respect to the symmetric part of the connection of the space 4 .

The Pole Ladder. We propose here a different construction for the parallel transport of vectors based on geodesics parallelograms. If the curve $C$ is geodesic then it can be itself one of the diagonals, and the Schild's ladder can therefore be adapted by requiring the computation of only one new diagonal of the parallelogram. We define in this way a different ladder scheme, that we name the "Pole Ladder" (Figure (2A)).

We now prove that the Pole ladder is actually realizing the parallel transport. In the diagram of Figure $(2 \mathrm{~A})$, the parallel transport of the tangent vector $v=\dot{C}$ to the geodesic $C$ is specified by the geodesic equation $\dot{\mathbf{v}}+\Gamma_{i j}^{k} \mathbf{v}^{i} \mathbf{v}^{j}=0$. In a sufficiently small neighborhood the relationships can be linearized to give

$$
\mathbf{v}^{k}(t)=\mathbf{v}^{k}(0)-t \Gamma_{i j}^{k}(x(0)) \mathbf{v}^{i}(0) \mathbf{v}^{j}(0)+O\left(t^{2}\right),
$$

and by integrating:

$$
x^{k}(t)=x^{k}(0)+\mathbf{v}^{k}(0)-\frac{t^{2}}{2} \Gamma_{i j}^{k}(x(0)) \mathbf{v}^{i}(0) \mathbf{v}^{j}(0)+O\left(t^{3}\right) .
$$

By renormalizing the length of the vector $v$ so that $C(-1)=P_{0}, C(0)=M$ and $C(1)=Q_{0}\left(\right.$ and denoting $\left.\Gamma_{i j}^{k}=\Gamma_{i j}^{k}(M)\right)$, we obtain the relations:

$$
\begin{aligned}
& P_{0}{ }^{k}=M^{k}-\mathbf{v}_{M}^{k}-\frac{1}{2} \Gamma_{i j}^{k} \mathbf{v}_{M}^{i} \mathbf{v}_{M}^{j}+O\left(\|\mathbf{v}\|^{3}\right), \\
& Q_{0}{ }^{k}=M^{k}+\mathbf{v}_{M}^{k}-\frac{1}{2} \Gamma_{i j}^{k} \mathbf{v}_{M}^{i} \mathbf{v}_{M}^{j}+O\left(\|\mathbf{v}\|^{3}\right) .
\end{aligned}
$$


Similarly, we have along the second geodesic:

$$
\begin{gathered}
P_{1}{ }^{k}=M^{k}-\mathbf{u}_{M}^{k}-\frac{1}{2} \Gamma_{i j}^{k} \mathbf{u}_{M}^{i} \mathbf{u}_{M}^{j}+O\left(\|\mathbf{u}\|^{3}\right), \\
Q_{1}{ }^{k}=M^{k}+\mathbf{u}_{M}^{k}-\frac{1}{2} \Gamma_{i j}^{k} \mathbf{u}_{M}^{i} \mathbf{u}_{M}^{j}+O\left(\|\mathbf{u}\|^{3}\right) .
\end{gathered}
$$

Now, to compute the geodesics joining $P_{0}$ to $P_{1}$ and $Q_{0}$ to $Q_{1}$, we have to used a Taylor expansion of the Christoffel symbols $\Gamma_{i j}^{k}$ around the point $M$. In the following, we indicate the coordinate according to which the quantity is derived by the index after a comma: $\Gamma_{i j, a}^{k}=\partial_{a} \Gamma_{i j}^{k}$ :

$$
\Gamma_{i j}^{k}\left(P_{0}\right)=\Gamma_{i j}^{k}+\Gamma_{i j, a}^{k}\left(-\mathbf{v}_{M}^{k}-\frac{1}{2} \Gamma_{i j}^{k} \mathbf{v}_{M}^{i} \mathbf{v}_{M}^{j}\right)+\frac{1}{2} \Gamma_{i j, a b}^{k} \mathbf{v}_{M}^{a} \mathbf{v}_{M}^{b}+O\left(\|\mathbf{v}\|^{3}\right)
$$

However, the Christoffel symbols are multiplied by a term of order $O\left(\|A\|^{2}\right)$, so that only the first term will be quadratic and all others will be of order 3 with respect to $A$ and $\mathbf{v}_{M}$. Thus, the geodesics joining $P_{0}$ to $P_{1}$ and $Q_{0}$ to $Q_{1}$ have equations:

$$
\begin{aligned}
& P_{1}^{k}=P_{0}^{k}+A^{k}-\frac{1}{2} \Gamma_{i j}^{k} A^{i} A^{j}+O\left(\left(\|A\|+\left\|\mathbf{v}_{M}\right\|\right)^{3}\right) \\
& Q_{1}^{k}=Q_{0}^{k}+B^{k}-\frac{1}{2} \Gamma_{i j}^{k} B^{i} B^{j}++O\left(\left(\|B\|+\left\|\mathbf{v}_{M}\right\|\right)^{3}\right)
\end{aligned}
$$

Equating $P_{1}^{k}$ in the previous equations gives

$$
\mathbf{u}_{M}^{k}+\frac{1}{2} \Gamma_{i j}^{k} \mathbf{u}_{M}^{i} \mathbf{u}_{M}^{j}=\mathbf{v}_{M}^{k}-A^{k}+\frac{1}{2} \Gamma_{i j}^{k}\left(\mathbf{v}_{M}^{i} \mathbf{v}_{M}^{j}+A^{i} A^{j}\right)+O\left(\left(\|B\|+\left\|\mathbf{v}_{M}\right\|\right)^{3}\right)
$$

Solving for $\mathbf{u}$ as a second order polynomial in $v_{M}$ and $A$ gives

$$
\mathbf{u}^{k}=\mathbf{v}_{M}^{k}-A^{k}+\frac{1}{2}\left(\Gamma_{i j}^{k}+\Gamma_{j i}^{k}\right) A^{i} \mathbf{v}_{M}^{j}+O\left(\left(\|A\|+\left\|\mathbf{v}_{M}\right\|\right)^{3}\right) .
$$

Now equating $Q_{1}^{k}$ in the previous equations gives

$$
B^{k}-\frac{1}{2} \Gamma_{i j}^{k} B^{i} B^{j}=-A^{k}+\left(\Gamma_{i j}^{k}+\Gamma_{j i}^{k}\right) A^{i} \mathbf{v}_{M}^{j}+\frac{1}{2} \Gamma_{i j}^{k} A^{i} A^{j}+O\left(\left(\|A\|+\left\|\mathbf{v}_{M}\right\|\right)^{3}\right) .
$$

Solving for $B^{k}$ as a second order polynomial in $v_{M}$ and $A$ gives:

$$
B^{k}=-A^{k}+\left(\Gamma_{i j}^{k}+\Gamma_{j i}^{k}\right) A^{i} \mathbf{v}^{j}+O\left(\left(\|A\|+\left\|\mathbf{v}_{M}\right\|\right)^{3}\right) .
$$

To verify that this is the correct formula for the parallel transport of $A$, let us observe that the field $A(x)$ is parallel in the direction of $\mathbf{v}^{j}$ if $\nabla_{V} A=0$, i.e. if $\partial_{v} A^{k}+\Gamma_{i j}^{k} A^{i} \mathbf{v}^{j}=0$, which means that $A^{k}(x+\epsilon v)=A^{k}-\epsilon \Gamma_{i j}^{k} A^{i} \mathbf{v}^{j}+O\left(\epsilon^{2}\right)$. This shows that the Pole ladder is realizing the parallel transport for a length $\epsilon=2$ (remember that our initial geodesic was defined from -1 to 1 ) if the connection is symmetric $\left(\Gamma_{i j}^{k}=\Gamma_{j i}^{k}\right)$.

We have thus demonstrated that the vector $-B$ of Figure $(2 \mathrm{~A})$ is the transport of $A$ and, due to the locally linear construction, it corresponds necessarily to the one transported by the Schild's Ladder. 


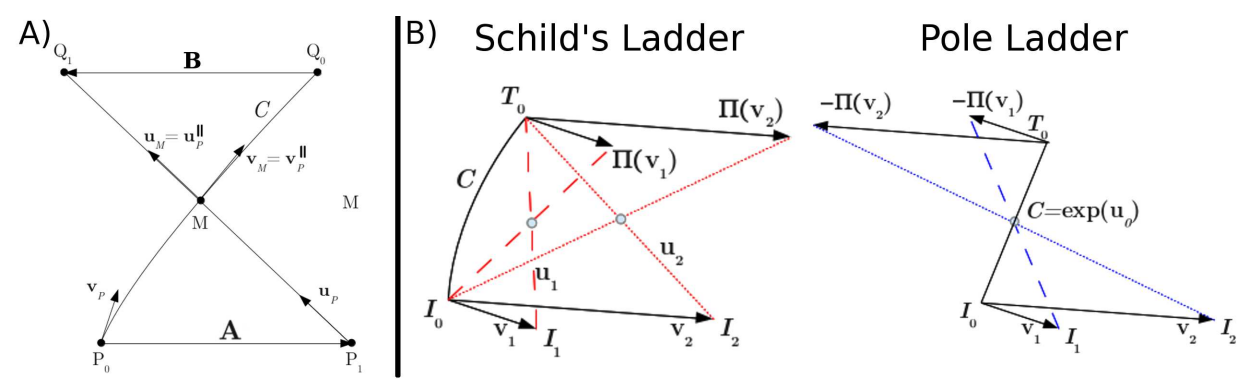

Fig. 2: A) The Pole ladder parallel transports the vector $\mathbf{A}$ along the geodesic $C$. Differently from the Schild's ladder it requires to compute only one diagonal geodesic from $P_{1}$ to $Q_{1}$. B) Geometrical schemes of the Schild's ladder and of the Pole ladder. By using the curve $C$ as diagonal, the Pole ladder requires the computation of half times of the geodesics (blue) required by the Schild's ladder (red).

\subsection{Application to Images}

Let $I_{i}(i=1 \ldots n)$ be a time series of images, and let $\varphi_{i}=\exp \left(v_{i}\right)$ the progression of longitudinal deformations from baseline to follow-up such that $I_{i}=\varphi_{i} * I_{0}$. Given a reference template $T_{0}$, let $u_{i}$ is the inter-subject SVF such that $T_{0}=$ $\exp \left(u_{i}\right) * I_{i}$. In [7] we showed that the Schild's Ladder for the transport of $v_{i}$ from $I_{0}$ to $T_{0}$ can be computed in closed form by the following formula:

$$
\Pi_{\text {Schild }}\left(v_{i}\right) \simeq v_{i}+\left[u_{i} / 2, v_{i}\right]+\frac{1}{2}\left[u_{i} / 2,\left[u_{i} / 2, v_{i}\right]\right],
$$

In case of time series of images over multiple time points, the Schild's ladder therefore requires the estimation of the series of inter-subject SVFs $u_{i}$. In addition to the computational problem of estimating several deformations through non-rigid registration, in this case the transport of the time series $v_{i}$ is not computed consistently with respect to the same inter-subject SVF. For this reason the resulting transported $\Pi\left(v_{i}\right)$ might be more biased by registration approximations.

The Pole ladder is similar to the Schild's one, with the difference of explicitly using as a diagonal the geodesic $C=\exp \left(u_{0}\right)$ which connects $I_{0}$ and $T_{0}$. This is an interesting property since, given $C$, the transport of time series of several images is based on the same baseline-to-reference SVF (Figure (2B)). Let $u_{0}$ the baseline-to-reference SVF, the Pole ladder is therefore computed in closed form in the following way:

$$
\Pi_{\text {Pole }}\left(v_{i}\right) \simeq-v_{i}-\left[u_{0} / 2, v_{i}\right]-\frac{1}{2}\left[u_{0} / 2,\left[u_{0} / 2, v_{i}\right]\right],
$$




\section{Experiment: Modeling One-Year Brain Changes in Alzheimer's Disease}

Images corresponding to the baseline $I_{0}$ and the one-year follow-up $I_{1}$ scans were selected for 135 subjects affected by Alzheimer's disease from the ADNI database. For each subject $i$, the pairs of scans were rigidly aligned, and the longitudinal changes were measured by non-rigid registration using the LCCDemons algorithm [68].

The resulting deformation fields $\varphi_{i}=\exp \left(v_{i}\right)$ were transported with the Pole Ladder (BCH scheme) in the template reference along the subject-to-template deformation. The group-wise longitudinal progression was modeled as the mean of the transported SVFs $v_{i}$. The areas of significant longitudinal changes were investigated by one-sample t-test on the group of log-Jacobian scalar maps of the transported deformations. For the sake of comparison, the one sample tstatistic was tested on the subject specific longitudinal log-Jacobian scalar maps transported into the template space along the subject-to-template deformation.

Figure 3 shows a detail from the mean SVF from the transported one-year longitudinal trajectories. The field flows outward from the ventricles to indicate a pronounced enlargement, an shows consistent contracting flow in the temporal areas. The same effect can be statistically quantified by evaluating the areas where the log-Jacobian maps are statistically different from zero. The statistical result is in agreement with the one provided by the simple scalar interpolation of the longitudinal log-Jacobian maps. However by parallel transporting we preserve also the multidimensional information of the SVFs.

\section{Conclusions and Perspectives}

In this study we proposed an improvement of the Schild's Ladder for the efficient transport of longitudinal deformations in a reference space from time series of images. In fact, the Pole Ladder preserves the simple mathematical formulation of the Schild's Ladder while optimizing the efficiency of the transport of series of longitudinal deformations. From the appliative point of view, the high spatial resolution of the statistical results of the experiment on the real data suggests a high precision of the procedure in transporting the different subject-specific trajectories. The Pole Ladder represents therefore a promising method for modeling the longitudinal changes in clinical populations by including multiple time points, and for comparing progressions of different clinical groups on a multidimensional basis.

\section{References}

1. Arsigny, V., Commowick, O., Pennec, X., Ayache, N.: A Log-Euclidean framework for statistics on diffeomorphisms. In: MICCAI. vol. 9, pp. 924-931 (2006)

2. Beg, M.F., Miller, M.I., Trouve, A., Younes, L.: Computing Large Deformation Metric Mappings via Geodesic Flows of Diffeomorphisms. Int. J. Comput. Vision 61(2), 139-157 (Feb 2005) 

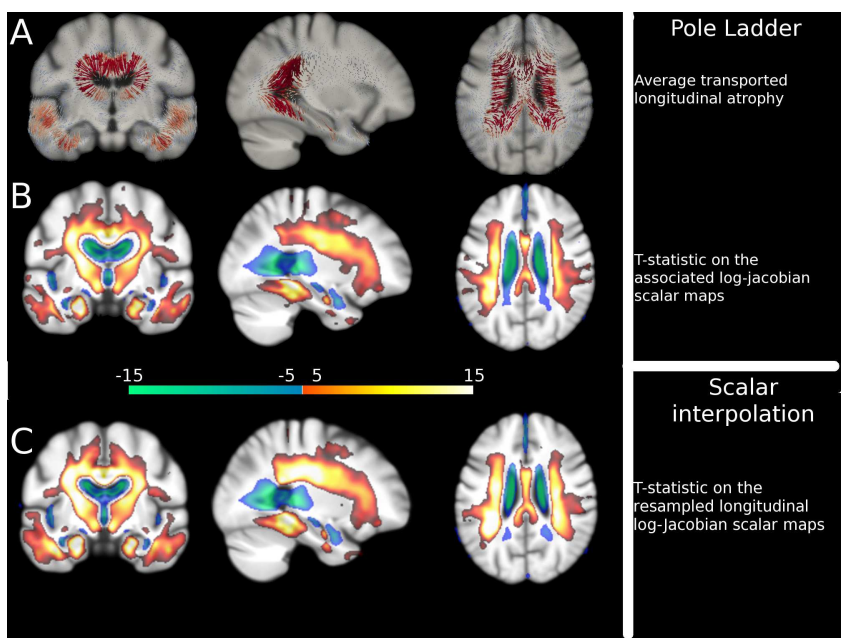

Fig. 3: One year structural changes for 135 Alzheimer's patients. A) Mean of the longitudinal SVFs transported in the template space with the Pole Ladder. B) T-statistic for the correspondent log-Jacobian values significantly different from $0(p<0.001$ FDR corrected). C) T-statistic for longitudinal log-Jacobian scalar maps resampled from the subject to the template space. Blue color: significant expansion, Red color: significant contraction.

3. do Carmo, M.: Riemannian Geometry. Mathematics, Birkhäuser, Boston, Basel, Berlin (1992)

4. Kheyfets, A., Miller, W., Newton, G.: Schild's Ladder parallel transport for an arbitrary connection. International Journal of Theoretical Physics 39(12), 41-56 (2000)

5. KSchild, A.: Tearing geometry to pieces: More on conformal geometry. unpublished lecture at Jan. 191970 Princeton Univesity relativity seminar (1970)

6. Lorenzi, M.: Deformation based morphometry of the brain for the development of surrogate markers in Alzheimer's disease. Ph.D. thesis, University orf Nice (2012)

7. Lorenzi, M., Ayache, N., Pennec, X.: Schild's Ladder for the parallel transport of deformations in time series of images. In: Information Processing in Medical Imaging - IPMI. vol. 22, pp. 463-474 (2011)

8. Lorenzi, M., Ayache, N., Frisoni, G., Pennec, X.: LCC-Demons: a robust and accurate diffeomorphic registration algorithm. NeuroImage (2013), to appear

9. Misner, C.W., Thorne, K.S., Wheeler, J.: Gravitation. W.H. Freeman and Compagny (1973)

10. Postnikov, M.M.: Geometry VI: Riemannian Geometry. Encyclopedia of mathematical science, Springer (2001)

11. Trouvé, A.: Diffeomorphisms groups and pattern matching in image analysis. Int. J. Comput. Vision 28(3), 213-221 (1998) 